\title{
Risk factors and Pathogenesis in the Development of Prosthetic ValveEndocarditis
}

\author{
Murtaza Mustafa.M.Phanindranath, Rajesh K Muniandy, M.Yusof Ibrahim, \\ Raihana Musawwir \\ Faculty of Medicine and Health Sciences, University Malaysia Sabah, Kota Kinabalu, Sabah,Malaysia.
}

\begin{abstract}
Mortality and morbidity in prosthetic valve endocarditis (PVE) is high despite improvements in diagnosis, therapeutic and design. Incidence of PVE is high in the initial 6 to 12 months after valve replacement and at low rate thereafter. Health care-associated infections are on the increase due to methicillin resistant Staphylococcus aureus,(MRSA), and coagulase negative staphylococci (CONS).Community acquired PVE is caused by enterococci, Streptococcus viridans group, and fastidious organisms, including the HACEK group. Microorganisms with surface components that react with adhesive matrix molecules (MSCRAMMs),mediate colonization and infection. Frequent risk factor for infection includes intravascular devices and hemodialysis. Antimicrobial therapy of PVE do not differ from native valve endocarditis (NVE), except larger vegetations than in NVE, must be considered.
\end{abstract}

KEYWORDS: Prosthetic valve endocarditis (PVE), Pathogenesis, Diagnosis, and Treatment.

\section{INTRODUCTION}

Prosthetic valve endocarditis (PVE) is associated with a high mortality during the early midterm follow-up despite diagnostic and therapeutic improvements; its incidence is increasing and reaches 20-30\% of all infective endocarditis episodes [1].Incidence of PVE is highest during the initial 6-to 12 months after valve replacement but continues at a low rate thereafter. By 4 to 5 years postoperatively as many as $3 \%$ to $6 \%$ of patients may have PVE.In most series, the rate of infection in mechanical and bio prosthetic valves is similar. The rate of infection are similar for prostheses at the mitral or aortic position [2].Incidence of PVE ranges from $1 \%$ to $6 \%$ of prosthetic valve placement ,or $0-.3 \%$ to $0.6 \%$ par patient-year [3].PVE accounts for $16 \%$ to $33 \%$ of all definitive cases of infective endocarditis, according to data from retrospective studies of single and multicenter tertiary care units in developed countries, and from prospective national, European and international multicenter observational studies[3-5]. Health care-associated infections are now the most important predisposition for the development of PVE worldwide [5].Across geographical regions, health care-associated infections are correlated with an increase in staphylococcal infections, including methicillin resistant Staphylococcus aureus (MRSA) and coagulase-negative staphylococci (CoNS).Staphylococci are the most common causative organisms of PVE.Major risk factors for infection include intravascular devices and hemodialysis. The rate of mortality from health care-associated PVE is high despite advanced diagnostic imaging and potentially curative medical and medical-surgical treatment approaches[5].Most community acquired PVE is caused by enterococci,Streptococcus viridans, group organisms, and fastidious organisms, including the HACEK group (Haemophilusspp, Actinobacillus actinomycetemcommitans, Cardiobacterium hominis, Eikenella corrodens, and Kingenella spp) and intracellular organisms[4].The basic principles of antimicrobial treatment in PVE do not differ from those for native valve endocarditis(NVE). Some special aspects need to be considered, however PVE is usually is associated with vegetations larger than those found in NVE[6].The paper reviews epidemiology, microbiology, diagnosis and therapy of PVE

\section{TIME OF ONSET AND FREQUENCY OF PVE}

PVE is an endovascular, microbial infection occurring on parts of a valve prosthesis or an reconstructed native heart valves[7].It is recommended to determine whether (a) mechanical prosthesis(b)a bioprosthetic xenograft stented or unstented(c)an allograft(d)a homograft,or(e)a repaired native valve with or without implantation of an annular ring is involved [8]. Although clinical relevance and therapeutic considerations may be similar, infections of devices or linens placed inside the heart but not connected to endocardial structures should be classified as "polymer associated infections "rather than PVE.PVE should be classified as either being acquired perioperatively(early PVE), or as community acquired (late PVE)[8].Because of significant differences in microbiology of PVE observed within the first year of operation and later on, the 
time cut off point between early and late PVE should be regarded as one year [9].The risk of early PVE is higher(approximately 5\%) in patients with replacement surgery during active infective endocarditis, especially if the casual organism is unknown or the antibiotic treatment is insufficient. The incidence of late PVE is lower for mechanical prostheses than for bioprotheses. The weighted mean incidence for infections of bioprotheses calculated from published series is $0.49 \%$ per patient per year for mitral valves and $0.91 \%$ per patient year for aortic valves.For mechanical prostheses the incidence is $0.18 \%$ per patient year for mitral,0.27\% per patient year for aortic,and0.29\% per patient year for multiple implants[10].

\section{PATHOGENESIS}

The thrombogenecity of the sewing cuff fabric of valve prostheses, sutures, annular and per annular mechanical and inflammatory lesions, and aging bioprothese favors deposition of fibrinogen fibrin, fibronectin, plasma proteins, and platelets that serve as targets for microorganisms with surface components that reacts with adhesive matrix molecules (MSCRAMMs) [11].These surface components mediate colonization and infection and/or modulate host defense mechanisms. Bacterial organisms that trigger infective endocarditis possess abundant MSCRAMMs. Only a limited number of these proteins have been assessed for their pathogenetic relevance in infective endocarditis [12]. S.aureus organisms display protein MSCRAMMs and non protein adhesins[13].The clumping factors a((CIfa),fibrigen binding-binding adhesions, and the bifunctional fibrinogen. fibronectin-binding protein A(FnBPA) are crucial in the pathogenesis of infective endocarditis[13].CIfA proteins are involved in early valve colonization and infection of the fibrin-platelet clot. In addition FnBPA promotes internalization by intact endothelial cells through a fibronectin bridge between FnBPA and endothelial $\alpha_{5} \beta_{1}$ integrins(fibronectin receptors).Intracellular S.aureus can lead to persistent or recurrent infection by providing host defense and membrane-active antimicrobial agents such as $\beta$-lactams and glycopeptides. The organisms replicates and lyse the endothelium by $\alpha$-hemolysin, thereby exposing the thrombogenic sub endothelial matrix, spreading into the circulation, invading adjacent endothelial cells, and producing a progressive infection. This can occur even in patients with structurally normal valves. Such complex behavior of S.aureus is coordinately regulated in growth phase gene regulator (Agr), the stress response regulon (SigB),and staphylococcal accessory regulator (SarA). These regulator genes sense environmental modification, trigger the secretion of tissue-degrading enzymes and toxins, and allow the bacteria to adapt and propagate[13].

Many MSCRAMMs for streptococci exist, as far as studied in experimental infective. endocarditisglucans(S.sanguis,Streptococcusmutans,Streptococcusgordonii);FimA (Streococcus parasanguis); Ace, a collagen adhesion for collagen type IV, collagen type I, and laminin,endocarditis, andbiofilimassociatedproteins(Enterococcusfaecalis) [14-16];Acm and Scm. collagen adhesins(Enterococcus faceium); Has, a sialic acid- binding protein (S.gordonii), and for Aggregatibacter actinomycetemcomitan, and EmaA.an oligomeric coiled-coil adhesion homologous to Yad A of Yesinia enterocolitis[17-19]. Adherent bacteria activate the extrinsic pathway of the clotting system by triggering release of tissue factor from monocytes that adhere to early vegetations, and from endothelial. Cells surrounding the infected valves [20,21].

S.aureus induces platelet activation by several surface proteins, CIfA/clumping factor B(CIB)and FnBPA/fibronectin-binding protein(FnBPA)are the major platelet activating modulins./The FnBPA/FnBPB and CIB proteins are expressed during experimental phase of growth[22]. S. sanguis strains differ in ability to adhere to and aggregate platelets [23].In strains that show strong adhesion and rapid platelet aggregation, activation in mediated by direct interaction between the serine-rich glycoprotein $\mathrm{A}(\operatorname{Srp} \mathrm{A})$ and glycoprotein $1 \mathrm{~b}$ receptor(von Willebr and factor[vWF])[24].For strains with long lag time activation requires specific antibody and complement assembly that link the surface proteins to platelet FcyR11A and complement receptors[25].Strains of S.gordonii stimulate aggregation directly by binding to glycoproteins $1 \mathrm{~b}$ an $11 \mathrm{~b}$ through serine-rich surface glycoproteins GspB and Sialic acid-binding adhesions(Has)[26].

Microorganisms inducing infective endocarditis have reduced susceptibility to platelet-microbicidal proteins in vitro [27].Fibrin adherent streptococci are not engulfed by monocytes. CIfA of S.aureus inhibits phagocytosis by human polymorph nuclear leukocytes in the absence of fibrinogen but showed enhanced inhibition in the presence of fibrinogen[28,29]. The experimental rat model of infective endocarditis S.gordonii strains are resistant to polymorph nuclear leukocytes killing after adhesion-mediated phagocytosis are more likely to cause infective endocarditis[30].

\section{PATHOPHYSIOLOGY}


Infection of mechanical prostheses starts at the interface between the sewing cuff and the native tissue. Depending on the virulence of the causative pathogen, infection can result in loosening of the sutures, which leads to periprosthetic leaks, or in ring abscesses, which may dislodge the prostheses from its anchorage and give rise to the echocardiographic appearance of a "dancing "prostheses. Ruptured ring abscesses may form fistulous tracks into surrounding tissues, extending the infection into cardiac chambers or into the intraventricular septum. Rupture may induce the development of intracardiac shunting. Infection extension to the aortic root may lead to the development of aneurysms of the sinus Valsalva. Infection extension from the aortic ring through mitral-aortic fibrous continuity may result in aneurysms on the anterior leaflet of the mitral valve. Valve incompetence with ventricular decomposition and congestive heart failure, heart block, systemic embolization of vegetations, and multiorgan infection with sepsis are noted complications [31].

In a retrospective multicenter study at 16 tertiary referral hospitals,148(17\%) of 872 patients with PVE had periannular complications the aortic ring, including aortocavitary fistulas in 19\% and non-ruptured abscess in $81 \%$.Patients with fistulas exhibited a sevenfold greater risk for developing concomitant ventricular septal defects and were more likely to develop heart failure or third-degree heart block than were patients with nonruptured abscess. Of all patients with PVE,45\% developed prosthetic dehiscence with moderate or severe valve regurgitation. Staphylococci were the most common causative microorganisms:S.aureus in 19\% and CoNS in $39 \%$.Mechanical valve(as opposed to bioprosthetic valves) were involved in $62 \%$ of the patients[32]

The sizes and types of vegetations are correlated with the virulence of the causative miroorganisms.S.aureus infections are mostly associated with small vegetations and extension of infection that are highly destructive of tissue. Streptococcal infections tend to larger vegetations with slower, milder destruction of tissue. Fungi form large, bulky vegetations. Large vegetations are suggestive of infection with one of the HACEK group of organisms[31]. With bioprosthetic valves, which are partly fabricated from porcine aortic valves or bovine pericardium, infection is restricted to the cusps, in contrast to mechanical prosthesis, in which valve materials are not well suited for bacterial adhesion. The growth of thrombotic vegetations in biosynthetic material can lead to cusp rupture, perforation, and vegatations. If sewing cuff in involved in infection, the pathologic process is similar to that of prosthetic valve infection. During reconstructive surgery of the mitral valve, a ring is often implanted from the arterial aspect for mobilization of the ring structure. The risk of infection is low. Whether mechanical or bioprosthetic valves are more prone to infection remains unresolved at present time [12].

\section{EARLY AND LATE ONSET OF PVE}

PVE is categorized as early-onset PVE (E0-PVE) and late onset (L0-PVE) on the basis of how time elapses between prosthetic valve replacement and onset to symptoms. There is no agreement in time designations. Some authors consider 60 days, less than 6 months or 1 year after valve replacement as E0-PVE and thereafter as L0-PVE [5,33,34].Some authors observed differences in the rates of bacterial microorganisms between E0-PVE and L0-PVE[5].During the late 1970s,gram-negative bacilli were rare causes of E0-PVE.The most frequent pathogens were CoNS and S.aureus[5].In L0-PVE,streptococci are surpassed by staphylococci as the most frequent causative organisms in some regions of the world. Methicillin- resistant organisms are more frequent in E0-PVE than in L0-PVE.The most common organisms isolated from L0-PVE are streptococci of S.viridans group and Enterococcus spp. In some university- affiliated hospitals, enterococci have surpassed S.viridan organisms and thus are the third most common etiologic agent of PVE[35].Group D streptococci are an emerging cause of infective endocarditis in some European countries particularly in France[4].For rural residents, the frequency of group D streptococcal infective endocarditis was twice as high as that of oral streptococci; it was also higher in mixed rural and urban populations than in urban regions[36]The disparity in the rank order of microbial causes of infective endocarditis between countries is great[36].The cumulative risk of developing PVE is highest within initial 12 months after replacement surgery, with peak during the first 2 months. During the time between implantation and complete endothelialization, patients are vulnerable to PVE. This period is also the time of more frequent health care contacts. One year after replacement would be a more reliablereference point to distinguish between E0-PVE and L0-PVE, especially in view of the wide range (4 to 328 days) between valve surgery and onset of symptoms of endocarditis[5,37].

\section{CLINICAL FEATURES}


The elapsed from infection to appearance of clinical symptoms of both E0-PVE and L0-PVE is determined by the virulence of the pathogen is modified by the immune status of the host. Clinical findings are often caused by the periannular extension of infection and embolic events [32].Fever is the most frequent manifestation( $73 \%$ to $92 \%$ ). Fever may not be present in the elderly patients, in patients given antimicrobials therapy before presentation, or in patients with Whipple's disease, and it may be low grade or intermittent in Qfever endocarditis[38,39]. Common clinical manifestations are heart failure (33\% to $70 \%)$ and a new or changing murmur( $43 \%$ ) caused by either valve dehiscence $(39 \%)$ or valve obstruction by vegetations or by both. New conduction abnormalities, especially atrioventricular conduction disturbances, demonstrate intraventricular progression of infection(26\%).Embolism(20\%to40\%)with cerebral stroke(23\%)and peripheral involvement(12\%), metastatic infections(9\%)with splenomegaly(20\%), and petechiae (10\%) are further complications of PVE .Cerebral emboli were shown to be symptomatic in 35\% and clinically silent in $30 \%$ of 60 patients with left-sided infective endocarditis. Causes of mortality are cardiogenic shock or septic shock with multiorgan failure [39].Infection with low-virulence organisms manifest with a sub-acute or chronic course. In affected patients, symptoms are often subtle, which make the diagnosis challenging [12].

Approximately $50 \%$ of patients with prosthetic valve and S.aureus bacteremia and approximately $40 \%$ with CoNS bacteremia develop definitive endocarditis [40].Risk is high even in the absence of persistent fever and persistent bacteremia[41]. The risk has been shown to be independent of the type of,location,or age of the prosthetic valve[41].Among patients with enterococcal bacteremia infective endocarditis was observed in $8 \%$ to $32 \%$ [42].Patients with a prosthetic valve who develop S.aureus, CoNS, or enterococcal bacteremia should be aggressively screened with transesophegeal endocardiography(TEE) for evidence of endocarditis [12].

\section{DIAGNOSTIC METHODS}

The revised Duke criteria should be used as the primary diagnostic scheme when PVE is suspected. They are used primarily on identification of the causative organisms and results of TEE, which reflects the total inflammation [42,12]. The sensitivity and specificity of the modified Duke criteria for the diagnosis of definite PVE are not yet defined. The Duke criteria should be used with caution in making diagnosis of PVE, if blood culture findings are negative and if TEE results are uncertain despite an otherwise compatible clinical manifestation [43,12].Diagnostic approach in PVE does not differ from that in NVE as both are systemic infections maintaining a continuous bacteremia. Hence the diagnosis is established if in addition to typical signs and symptoms and positive blood culture, the device can be shown to be affected by echocardiography, preferably using multiplane transoesophegeal (TOE) probes.TOE should be performed without delay in all patients with suspicion of PVE[44].For the diagnosis of PVE,TOE is of such immense importance that institutions without this facility are best advised to ask assistance from a specialized Centre. With TOE the size of vegetation can be identified more precisely than with TEE, and periannular complications indicating a locally uncontrolled infection(for example abscesses, dehiscence,fistulas) may be detected earlier. Both size of vegetations and infection morphology significantly influence therapeutic decisions(name duration of antimicrobial treatment and the need for urgent surgical intervention).In otherwise unproven cases,gallium-67 scans or indium-111 leukocyte scintigraphy have reported to be useful in detecting myocardial abscesses or diffuse infiltration.Their diagnostic impact has not been established so far[45]. The broad- range bacterial ribosomal DNA(rDNA) gene polymerase chain reaction(PCR),followed by sequencing of either or both of the partially amplified $16 \mathrm{~S}$ and $23 \mathrm{~S}$ rDNA genes, is especially useful when the causative agent is fastidious or unculturable [46].

\section{TREATMENT}

Antimicrobial therapy. The basic principles of antimicrobial treatment in PVE do not differ from those for NVE. Some special aspects need to be considered however PVE is usually associated with vegetations larger than those found in NVE .Consequently antibiotics have to be used in dosages which result in maximum, on-toxic serum concentrations in order to penetrate the total vegetation. The duration of treatment usually has to be longer than for the treatment of NVE and should consider vegetation size as determined by TOE as well as the minimal inhibitory concentration(MIC) of the most efficient combination of antibiotics. Antibiotic sterilization of large vegetations is likely with an $\mathrm{MIC}>4 \mu \mathrm{g} / \mathrm{ml}$.Duration of antimicrobial treatment in prosthetic valve endocarditis with respect to vegetation size and MIC in PVE caused by coagulase-negative staphylococci complex interaction between the microorganism and the synthetic material-for example irreversible adhesion and production of biofilm which inhibit the host defense mechanism-protects against antimicrobial treatment and makes antibiotic sterilization extremely difficult $[47,6]$. 
The presence of (micro) abscesses is likely in PVE caused by coagulase-negative staphylococci, and triple therapy including rifampicin $(900 \mathrm{mg} /$ day divided into three doses) is recommended[48].Rifampicin is actively taken up by granulocytes and becomes effective against intracellular staphylococci and staphylococci inside the abscesses [49]. When PVE is clinically apparent and blood cultures are not yet positive empiric treatment should be initiated with vancomycin and gentamicin[8].

Antithrombotic treatment. Antithrombotic treatment in patients with PVE has been discussed widely. There seems to be a consensus that oral anticoagulants treatment should be suspended and replaced by intravenous heparin. The dosage of heparin depends on the presence of secondary complications (for example thrombocytopenia) and varies between $7-430 \mathrm{U} / \mathrm{kg}$ body weight. Low molecular weight heparins mayadvantageous, as side effects (especially thrombocytopenia) are less frequent [50].

Surgical intervention. If PVE is complicated, it has to be decided whether medical treatment should be continued or urgent surgical intervention is required. The indications for surgery in PVE are similar to those in NVE large (>10mm) mobile vegetarians thrombolic events with vegetations, sepsis persisting for more than 48 hours despite effective antibiotic treatment(guided by blood cultures and MICs)and acute renal failure. A cerebral embolic event is not a contraindication for open heart surgery provided that there is no cerebral hemorrhage and the time between embolic event and surgery is short ( $<72$ hours)so that the blood- brain barrier can be expected not to be significantly disturbed[51]. Periprosthetic dehiscence with or without myocardial failure has a poor prognosis. If congestion is not promptly removed by medical treatment, surgical intervention is mandatory. Allograft aortic root replacement is valuable technique in the complex setting of PVE with involvement of the periannular region [52]

Mortality in PVE. Prognostic factors identified by multivariate analysis is independently associated with higher mortality rate [5].These factors have been completely or partially confirmed by several groups[34,35].The type of valve, the position of the prosthesis, and time of onset of infection have not been identified as independent factors to mortality $[5,35]$.

\section{CONCLUSION}

High incidence of PVE within first year of valve replacement.PVE is a frequent indication for surgery. Treatment for PVE and NVE is the same, although PVE have larger vegetations.

\section{REFERENCES}

[1]. 1.Nataloni M,Pergolini M,Rescigno G,et al.Perosthetic valve endocarditis.J.CarvascMed.2010;11(12):869-83.

[2]. Karchmer AW.Infections of prosthetic heart valve,In:Bisno AL,Waldvogel FA, eds.Infections associated with indewelling medical devices, $3^{\text {rd }}$ Ed. Washington, DC:American Society for Microbiolofy,2000;145-172.

[3]. Akowuah EF,Davies W,Oliver S,et al.Prosthetic valve endocarditis early and late outcome following medical or surgical treatment.Heart.2003;89:269-72.

[4]. Hoen B,Alla F,Selton Suty C;Association pour I'Etrude et la Prevention de I;Endocardite Infectience(AEPE)Study Group.Changing profile of infective endocarditis results of a 1 -year survey in France.JAMA.2002;288(1):75-81.

[5]. Wang A,Athan E,Pappas PA,et al.International Collaboration on endocarditis-Prospective Cohort Study Investigations. Contemporary clinical profile and outcome of prosthetic valve endocarditis.JAMA.2007;297:1353-61.

[6]. Hyde JAJ,Daroulche R0,Costeron JW.Strategies to prophylaxis against prosthetic valve endocarditis.J.Heart Valve Dis.1998;7:316-26.

[7]. Edmunds LH,Clark RE,Cohn IH,et al.Guidelines for reporting morbidity and mortality after cardiac vascular operation.Ann Thorac Surg.1988;48:257-59.

[8]. Horstkotte D,Follath F,v Graevenitz A, et al.Recommendations for prevention, diagnosis and treatment of infective endocarditis.The task force on infective endocarditis of the European Society of Cardiology.Eur Heart J.( in press \}.

[9]. Karchmer AW,Gibbons GW.Infections of prosthetic heart valves and vascular grafts.Ininfections associated with indwelling medical devices.eds Bisno AL.Waldvogel FA(ASM Press. Washington).1994;213-49.

[10]. Horstkotte D,Piper C,Niehues R, et al.Late prosthetic valve endocarditis.Eur HeartJ.1995;16(SupplB \} 39-47.

[11]. PatiJM,Allen BI,McGavin MJ,et al.MSCRMM-mediated adherence of microorganisms to host tissue.[Review].Annu Rev Microbiol.1994;48:585-617.

[12]. Knoll BM.Baddour LM,Wilson WR.Prosthetic valve Endocarditis.In Mandell,Dougllas andBennett's Principles and Practice of Infectious Diseases, $7^{\text {th }}$ ed.Mandell GI,Bennett JE,Dolin R(editors).Churchill Livingstone Elsvier,2010.

[13]. Massey RC,Kantzanon MN,Fowler T,et al,Fibronectin-binding protein A of htaphylococcusaureus has multiple substituting,binding regions that mediate adherence in fibronetin and invasion of endothelial cells.Cell MIcrobiol.2001;5:839-51.

[14]. Munro CI,Macrina FI.Sucrose-derived exopolysaccharides of Streotococcus mutans V403 contribute to infectivity in endocarditis.Mol Microbiol.1993;8(1):133-42.

[15]. Bornette Curtly D, Wells V, Viscount H,et al.FimA.a major virulence factor associated withStreptococcus parasnaguis endocarditis.Infect Immun.1995;63:4669-74.

[16]. Nallapareddy SR,Qin X,Weinstock GM,et al.Enterococcus faecalis adhesion,ace mediates attachment to extracellular matrix proteins collagen type IV and laminin as well collagen type 1.Infect Immuno.2000;68:5218-24. 
[17]. Nallapareddy SR,Singh KV,Murray BE.Contribution of the collagen adhesion Acm to pathogenesis od Enterococcus faceium in experimental endocarditis.Infect Immun. 2008;76:4120-28.

[18]. Urano-Tashiro Y,Yajima A,Takshima E,et al.Binding of the Streptococcus gordonii DI.1 surface protein Has to the host cell membrane glycoproteins CD11b,CD43 and CD50,.Infect Immun.2008;76:4686-91.

[19]. Tang G,Kitten T,Munro CI,et al.EmaA,a potential virulence determinant of Aggregatibacter actinomycetemocommitan in infective endocarditis.Infect Immun .2008;76:2316-24.

[20]. Bancsi MJ,Veltrop MH,Berina RM,et al.Influence of monocytes and antibiotic treatment on tissue factor activity of endocardial vegetations in rabbits infected with Streptococcus sanguis.Infet Immun.1996;64:448-451.

[21]. Yeh CY,Chen JY,Chua JS.Glucosyltransferase of viridans group streptococci modulate interleukin-6 and adhesion molecule expression in endothelial cells and augment monocytic cell adherence. Infect Immun.2006;74:1273-1283

[22]. Saravia-Otten P,Muller HP,Arvidson S.Transcription of Staphylococcus aureus fibronectin-binding protein genes is negatively regulated by Agr andAgr-independent mechanism.J Bacteriol.1997;179:5259-63.

[23]. Kerringan SW,Jakubovics Ns,,Keane C,et al.Role of Streptococcus gordonii surface protein SspA/SspB and Has in platelet function. Infect Immun.2007;75:5740-47.

[24]. Plummer C,Wu H,Kerringan SW,et al.A serine-rich glycoprotein ofStreptococcus sanguis mediates adhesion to platelet via GPIb.Br J Haematol.2005;129(1):101=109.

[25]. Ford J,Douglas CW.The vrole of platelets in infective endocarditis .Platelets. 1997;8:285-94.

[26]. Takahshi Y,Yajima A,Cisar J0,et al.Functional analysis of the Streptococcus gordonii DI.I sialic acid-binding adhesion and its essential role in bacterial binding to platelets. Infect Immun.2004;72:3876-3882.

[27]. Bayer AS,Cheng D,Yeaman MR,et al.In vitro resistance to thrombin-induced platelet microbicidal protein among clinical bacteremic isolates of Staphylococcus aureus correlates with a endovascular infections source.Antimicrob Agents Chemother. 1998;42:3169-72.

[28]. Bancsi MJ,Weltrop MH,Bertina RM,et al.Role of phagocytosis in activation of coagulation system in Streptococcus sanguis endocarditis.Infect Immun. 1996; 64: 5166-70.

[29]. Higgins J,Loughman A,van Kessel KP,et al.Clumping factor of Staphylococcus aureusinhibits phagocytosis by human polymorphonuclear leukocytes. FEMS Microbiol Lett.2006;258:290-96.

[30]. Young Lee S,Cisar J0,Bryant JI,et al.Resistance of Streotococcus gordonii to polymorphonucler leukocyte killing is a potential virulent determinant of infective endocarditis.Infect Immun.2006;74:5148-55.

[31]. Jegatheeswaran A,Butany J.Pathology of infection and inflammatory diseases in prosthetic heart valves. Cardiovasc Pathol.2006;15:252-55.

[32]. Anguera I,Miro JM,San Roman, et al.Periannular complications in infective endocarditis involving prosthetic aortic valves.Am $J$ Cardiol.2006;98:1261-68.

[33]. Tornos P,Iung R,Permanyer-Miralda G,et al.Infective endocarditis in Europe: lessons from the Euro heart survey.Heart.2005;91:510-75.

[34]. Lopez J,Revilla A,Vilacosta I,et al.Definition clinical profile microbiological spectrum, and prognostic factors of early onset of prosthetic valve endocarditis. Eur HeartJ.2007;28:760-65.

[35]. Rivas P,Alonso J,Moya J,et al.The impact of hospital -acquired infections on the microbial etiology and prognosis of late-onset prosthetic valve endocarditis. Chest. 2005;128:764-71

[36]. Giannisioti F,Chirouze C,Bouvet A,et al.AEPEI Study Group. Characteristics and regional variations of group D streptococcal endocarditis in France.Clin MicrobiolInfect.2007;13:770-76.

[37]. Fernandez-Guerrero MI,HerreroI,RellverM,etal.Nosocomial enterococcal endocarditis:a serious hazard for hospitalized patients with enterococcal bacteremiaJ Int Med.2002;252:510-15.

[38]. Anugera I,Miro JM,Cabell CH,et al.ICE-MD Investigators.Clinical characteristics and outcome of aortic endocarditis with periannular abscess in the international collaboration on Endocarditis Merged Database.Am J Cardiol.2005;96:976-81.

[39]. Snygg-Marin U,Gustafsson I,Rosengren I, et al.Cerebrovascular complications in patients with left- sided infective endocarditis are common: a prospective study using magnetic resonance imaging and neurochemical brain damage markers.Clin InfectDis.2008;47(1):23-30.

[40]. El-Ahdab F,Benjamin DK, Jr,Wang A,et al Risk of endocarditis among patients with prosthetic valves and Staphylococcus aureus bacteremia.Am J M.2005;118:225-29.

[41]. Fowler VG, Jr,Li J.Corey GR,et al.Role of echocardiography in evaluation of patients with Staphylococcus aureus bacteremia experience in 103 patients.J Am CollCaridol.1997;30:1072-78.

[42]. Anderson DJ,Olaison I,McDonald JR,et al.Enterococcal prosthetic valve infective endocarditis reportof45 episodes from the international Collaboration on Endocarditis-merged database.Eur I Clin Microbiol Infect Dis.2005;24:665-70.

[43]. Li JS, et al.Proposed modifications to Duke-criteria for the diagnosis of infective endocarditis.Clin Infect Dis.2000;30:633-38.

[44]. PedersenWR, WalkerM,OlseonJD, etal. Valueofthe transesophegeal endocardiography in evaluation of native valve endocarditis. Chest.1991;100:351-56.

[45]. 0'Brien K,Barnes D,Martin RH,et al.Gallium-SPECT in the detection of prosthetic valve endocarditis and aortic ring abscess.J Nucl Med.1991;32:1791-93.

[46]. Bosshard PR,Kronenberg A,Zhinden R,et al.Etiologic diagnosis of infective endocarditis by broad-range polymerase chain reaction: a 3 year experience. Clin Infect Dis.2003;37:167-72

[47]. Horstkotte D, Weist K,Rueden Better understanding of the pathogenesis of prosthetic valve endocarditis-recent perspectives for prevention strategies.Heart Valve Dis. 1998;7:313-15.

[48]. Wilson Wr,Geraci JE,Danielson GU,et al.Anticoagulant therapy and central nervous system complications in patients with prosthetic valve endocarditis. Circulation.1978;57:1004-7. 
[49]. Zimmmerli W.Experimental models in the investigation of device-related infections.J Antimicrob Chemother.1993;31(Suppl D)97102

[50]. Wilson WR,Karchmer AW,Dajani AS,et al.Antibiotic treatment of adults with infective endocarditisduetostreptococci, enterococci,staphylococci and HACEK microorganisms. JAMA.1995;274:1706-13.

[51]. Horstkotte D,Piper CWiemer M,et al.Dringlicher Herzklappenersatz nach acuter Himembolie wahrend florider Endokarditis.Med.Klinik.1998;93:284-93.

[52]. Dossche KM,Defauw JJ,Emst SM,et al.Allograft aortic root replacement in prosthetic aortic valve endocarditis a review of 32 patients.Ann Thorac Surg.1997;63:1644-49. 